\title{
Polarimetry of a sunspot at disk centre
}

\author{
M. Sánchez Cuberes ${ }^{1}$, K. G. Puschmann ${ }^{2}$ and E. Wiehr ${ }^{2}$ \\ ${ }^{1}$ Astrophysikalisches Institut Potsdam, Germany; email: msanchez@aip.de \\ ${ }^{2}$ Universitäts-Sternwarte, Germany; email: kgp@uni-sw.gwdg.de, ewiehr@uni-sw.gwdg.de
}

\section{Stokes data}

We observed a sunspot almost exactly at disc centre on 23 September, 2003, with the Tenerife Infrared Polarimeter (TIP; Martínez Pillet et al. 1999) at the $\phi=70 \mathrm{~cm}$ Vacuum Tower Telescope (VTT) on Tenerife. The highly symmetric spot passed the central meridian just at the time of observations; its solar latitude of $9^{\circ} \mathrm{N}$ was almost perfectly compensated by the inclination of the solar axis $B_{0}=7^{\circ}$. We took the full Stokes vector through the line profiles of Fer10783 $\AA$, Si I 10784.5 $\AA$ and Si $10786.8 \AA$. The spatial sampling was adapted to the pixel size of 0.4 arcsec width; the VTT correlation tracker largely compensated image motion. The Stokes spectra were corrected for the dark and the gain matrices, as well as for instrumental polarisation and Stokes cross-talk. The spectral resolution finally achieved with TIP and its detector amounts to $32 \mathrm{~m} \AA$.

The spatial distribution of the spectrally integrated I, Q, U, and V profiles (Fig. 1) shows a conspicuously high axial symmetry with respect to the sunspot centre: Stokes $\mathrm{V}$ decreases radially, Stokes - Q and - U increase with distance from the spot centre and exhibit a large azimuthal asymmetry. Inside the spot the Stokes profiles show the known shapes without sign reversals or anomalous polarisation profiles. However, in a ring outside the sunspot border, we find anomalous three-lobed Stokes - V profiles. These seem to be caused by two magnetic field components of opposite polarity in the same resolution element. In the anomalous $\mathrm{V}$-profiles, the components of opposite sign as the sunspot Stokes - V are significantly red-shifted. These may indicate 'returning flux' tubes which carry (at least a part of) the Evershed flow back to solar subsurface layers.

\section{Inversion results}

The finally obtained profiles have been inverted by means of the SIR code (Stokes Inversion based on Response function; Ruiz Cobo \& del Toro Iniesta 1992). This code yields for each pixel, the depth stratification of temperature $(\mathrm{T})$, magnetic field strength $(\mathrm{B})$, field inclination $(\gamma)$, field azimuth $(\phi)$, line-of-sight velocity $(\mathrm{v})$, and single values for micro- and macro-turbulence, both assumed to be constant with depth. We performed the inversion assuming a single magnetic component filling each resolution element, since at the very disc centre a two component inversion yields almost zero filling factors for one component. This may be due to our purely vertical view through largely horizontal Evershed flow channels, which hardly yields a radiative interlace of fine-structure atmospheres with significant relative Doppler shift.

Because of the almost perfect disk centre position of our spot, we do not need to make any assumption about geometric projection effects of field or flow. For the deep layers we find strong down-flows in the outer penumbra which increase with radial distance. In the inner penumbra, a 'bright ring' occurs where the line core intensities exceed those in the outer penumbra. Our inversion indicates a thermal origin for this feature. The spatial variation of the field inclination in Fig. 2 shows some fine-structure with stronger fields 


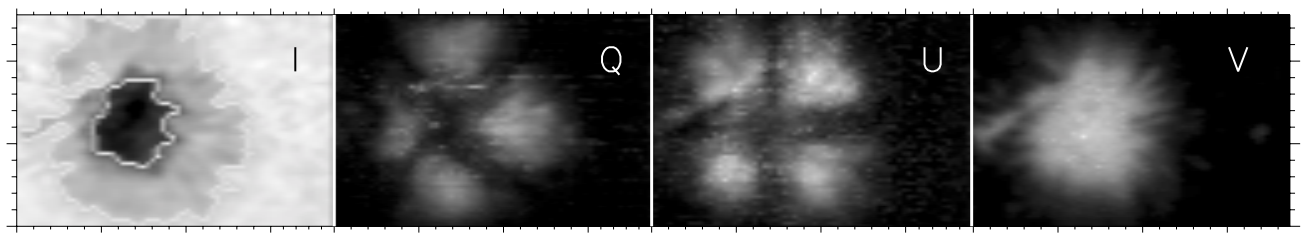

Figure 1. Two-dimensional presentation of the absolute values of Stokes-I, - Q, - U, and - V profiles integrated over the whole observed wavelength range covering the lines Fe I $10783 \AA$ Si I $10784.5 \AA$ and Si I $10786.8 \AA$; tick mark distance 2 arcsec.
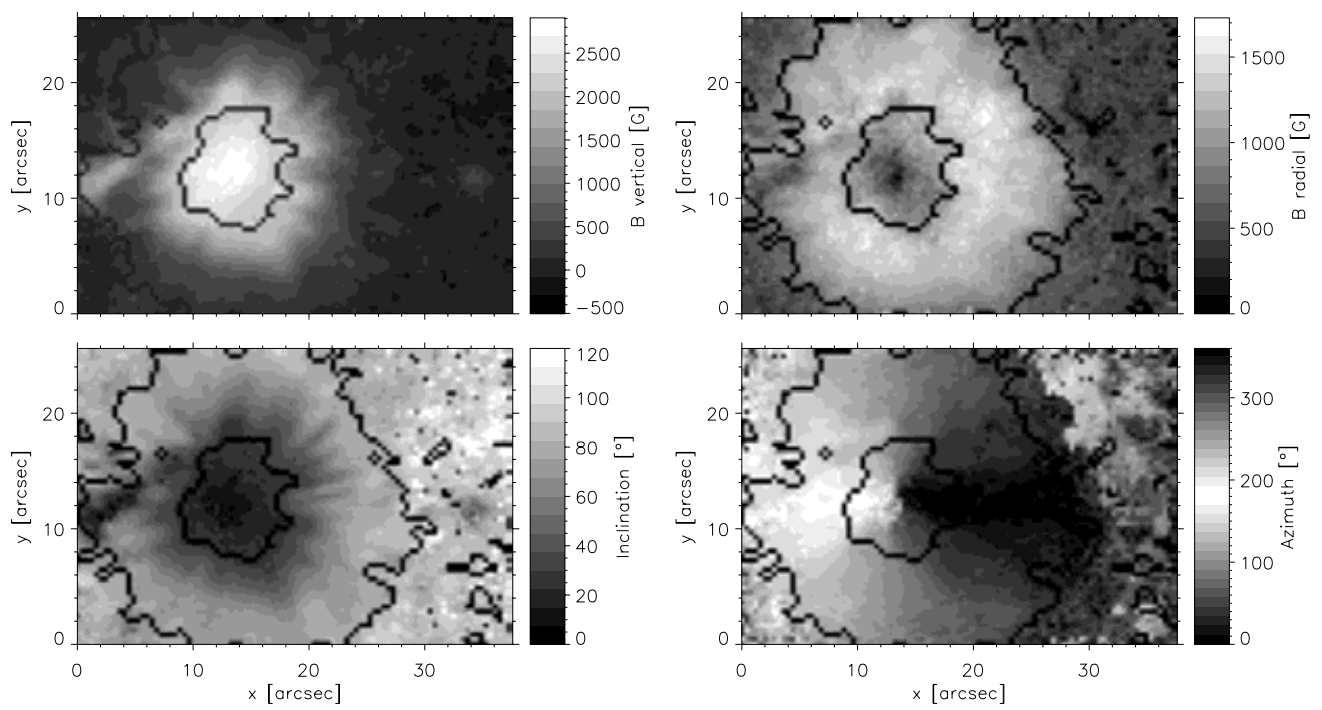

Figure 2. Two-dimensional presentation of the magnetic field parameters obtained at $\log \tau=0$ : vertical (upper left) and radial component (upper right), field inclination (lower left) and field azimuth (lower right panel) for the symmetric sunspot located almost perfectly at disk centre.

being more vertical, which is at variance with earlier findings (Beckers \& Schröter 1969; Schmidt \& Balthasar 1994; Wiehr 2000). A more detailed presentation of the results, in particular those from a two component inversion of spatial points showing anomalous Stokes profiles, will be published in a forthcoming paper.

\section{Acknowledgements}

We thank Dr. M. Collados Vera for his kind help and H. Balthasar for fruitful discussions; M. S. C. is supported by the 'European Solar Magnetometry Network' project of the European Commission under contract HPRN-CT-2002-00313. K. G. P. acknowledges support by the 'Deutsche Forschungsgemeinschaft' through grant KN 152/29-1.

\section{References}

Beckers, J. M. \& Schröter, E. H. 1969 Solar Phys. 10, 384.

Martínez Pillet, V., Collados, M., Sánchez Almeida, J. et al. 1999 ASP Conf. Series 118, 212.

Ruiz Cobo, B. \& del Toro Iniesta, J. C. 1992 ApJ 398, 375.

Schmidt, W. \& Balthasar, H. 1994 A\&A 283, 241.

Wiehr, E. 2000 Solar Phys. 196, 357. 